\title{
Energy saving at work - and when not working! Insights from a comparative study
}

\author{
Timo Jakobi \\ Workgroup on Human Computer Interaction \\ University of Siegen \\ Siegen, Germany \\ timo.jakobi@uni-siegen.de
}

\author{
Gunnar Stevens \\ Information Systems \\ Bonn-Rhein-Sieg University of Applied Science \\ Sankt Augustin, Germany \\ gunnar.stevens@uni-siegen.de
}

\begin{abstract}
Reducing energy consumption is one of the most pursued economic and ecologic challenges concerning societies as a whole, individuals and organizations alike. While politics start taking measures for energy turnaround and smart home energy monitors are becoming popular, few studies have touched on sustainability in office environments so far, though they account for almost every second workplace in modern economics. In this paper, we present findings of two parallel studies in an organizational context using behavioral change oriented strategies to raise energy awareness. Next to demonstrating potentials, it shows that energy feedback needs must fit to the local organizational context to succeed and should consider typical work patterns to foster accountability of consumption.
\end{abstract}

Keywords: Eco-feedback; Sustainable Interaction Design; Workplace; Organizational Culture

\section{INTRODUCTION}

In recent years Sustainable Interaction Design [6] has become a vivid research field in HCI. But compared to growing body of research on domestic energy use [10,16,17,28,34,40], significantly less research has yet investigated in behavior based energy saving potentials in office context [15,25,34]. Today, common approaches to reduce energy costs in organizations are mainly focused on technology. For example, Green IT research [24,26] investigates in concepts such as energy-efficient hardware and algorithms, green data center design, server virtualization and automated power management. Further technologically oriented measures are IT-enabled energy-efficient building- and applianceautomation [3,42]. For instance, smart solutions for HVAC and light control can help to reduce energy consumption, without employees being affected in their routines.

In order to tap into the full saving potential, however, technological rearrangements should be supplemented by behavioral measures known from Sustainable Interaction Design research in domestic context $[6,12,16]$. Still, as organizational context is different, it remains an open research question what usable and helpful design guidelines are, when

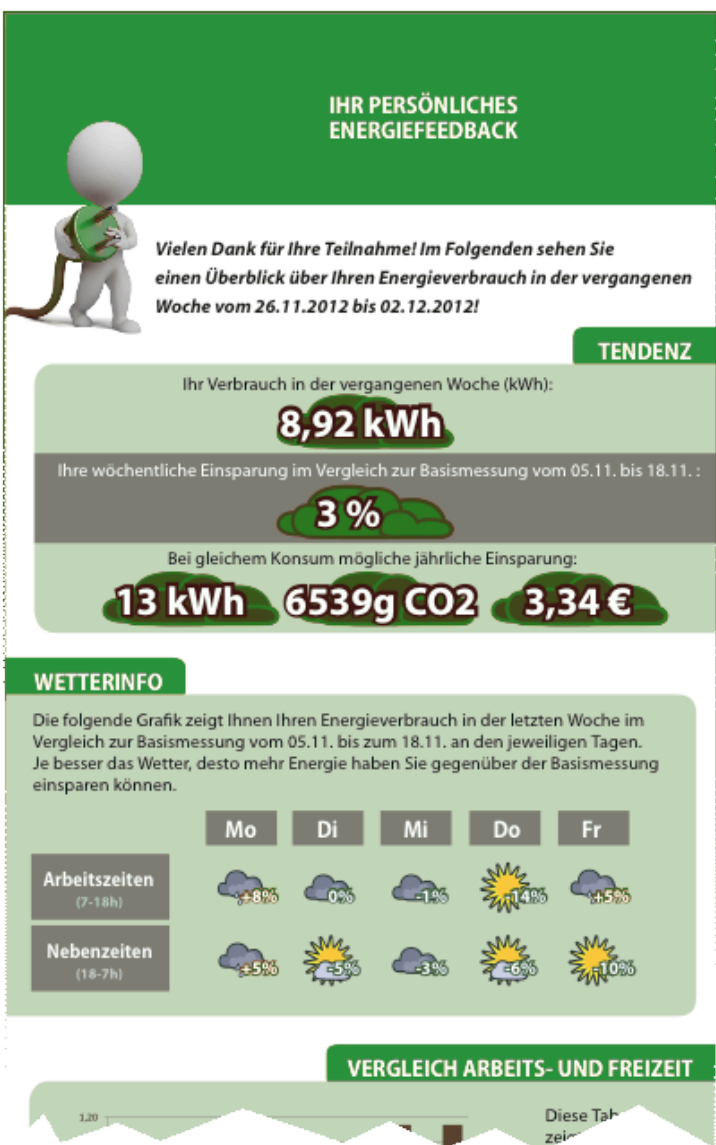

Figure 1: Part of a personalized feedback for office worker featuring overall consumption, relative comparison to last week, different reference systems and a weather metaphor

visualizing energy consumption at work, and in how far they might be transferred from established best practices in the home context.

Contributing to this gap, we conducted two studies, deploying smart sensors in organizations to measure energy consumption 
on device level. At both sites we ran a one-week information campaign and measured follow-up effects. Additionally, in the second company we explored design of personalized feedback reporting (cf. Figure 1) and studied its effects on consumption.

\section{BEHAVORIAL ENERGY SAVING IN THE OFFICE}

\section{A. Behavioral Environmental Research}

Behavioral environmental research got a first boost during the oil crisis in the 1970's [31]. Around that time, research of ecologic psychologists started to take an interest into the influence of behavior on energy consumption [38] and investigated into consumption feedback as a means of encouraging energy conservation [7]. Alongside, a body of theoretical approaches emerged within environmental research, seeking to understand individual's (un-)sustainable behaviors [16,20,39].

One of the most influential approaches roots in rational choice theory $[1,20]$ arguing that energy relevant behavior is steered by active and informed decision-making of consumers, who weigh pros and cons such as satisfaction of needs and costs and thus act according to their judgment. Other theories (like Stern's Value Belief Norm Theory [39]) also consider subjective norms, beliefs, and the influence of social surrounding.

By large, such concepts of norms and rational behavior ground persuasion and feedback campaigns theoretically $[13,16]$. In particular, design concepts such as providing direct feedback, enabling social comparison and supporting goal setting were inspired by these theories [2].

\section{B. Design for Behavioral Change}

Technological innovations have opened new opportunities to promote pro-environmental behavior and pushed novel solutions into the market $[11,37]$. In particular, Sustainable Interaction Design (SID) has become an established subfield of HCI, challenging the immateriality of energy [29] and exploring how consumption could be made visible and meaningful [7].

The spectrum of feedback design has become very wide, reaching from artistic solutions like the Power Aware Cord [17], over pragmatic ones like Watt-Lite [31], social norm oriented approaches [14,27] to HEMS integrating multiple features into a home-oriented system of services [34]. In addition, several empirical studies also demonstrate the effectiveness of eco-feedback in not only raising awareness but empowering consumers to implement savings, as well $[10,11,30,34]$. Yet, feedback systems are mainly studied in the domestic context.

\section{Energy Conservation of Office Workers}

In terms of designing interventions for organizational settings, there are only a few guidelines to design and conduct behavioral change oriented eco-campaigns.

Matthies et al. [21,23], for instance, have developed a tailored program including checklists, HOW-TOs, sample templates etc. to conduct eco-campaigns in buildings of public administration. With emphasis on companies and enterprises, the German Agency for Energy Efficiency has developed a similar campaign toolbox called MissionE1. Both mainly focus on classic materials like posters, flyers, information brochures and letters from superior authority. They also give some advice on how to use emails and websites, but suggestions for using smart technologies have yet to be integrated in these toolboxes.

Azar \& Menassa [4] have investigated into the design of organizational eco-feedback. They developed a decision support framework for conducting energy saving measures in commercial buildings. First general design guidelines and wireframe sketches were developed by Foster et al. [15] using focus group sessions. Based on a literature review about techniques of intervention appropriate for the workplace, Yun $[43,44]$ implemented a first functional prototype of an energydashboard.

The few studies evaluating eco-feedback in organizational context show mixed results. Carrico and Riemer [8] show that providing monthly feedback with a motivating message has a positive effect on energy saving of university employees. Installing eco-feedback applications on the desktop of university employees, Murtagh et al. [25] also found a significant reduction of consumption. However, they noticed a complex relationship between feedback and behavior and found a manifold of reasons exist 'not to switch things off'. Using smart metering technology in a research institute, Schwartz et al. [33] observed significant positive effects too, but only on short-term, with conservation fading successively over time.

Another issue addresses the question, which intervention strategies could and should be applied, in organizational context. For instance, in the domestic setting comparative feedback is a well-established strategy [16]. The study of Siero et al. [36] indicates that it can contribute to save energy in organizational settings, as well. Yet, we will show below, that this strategy cannot be transferred fully as it raises conflicts with privacy issues as well as values and norms of local organizational culture. This issue is already indicated by Schwartz et al. [33], who noted that some employees they interviewed are quite strict in showing their personal

${ }^{1}$ http://www.energieagentur.nrw.de/_database/_data/datainfop ool/mission_E_Kompendium_Leseprobe.pdf (9/6/13) 
consumption to colleagues or other parts of the organization, because they were afraid of misinterpretation or misuse.

\section{METHOD}

In order to explore potentials of behavioral energy saving measures, we investigated into the effects of information and feedback campaigns in two companies:

\section{A. Company Alpha}

Alpha is a global player in IT with facilities in more than 50 countries. The site taking part in our study works on Sustainable IT and has about 270 employees, mainly working in office environments. Company's culture can be characterized by low hierarchies, openness for innovation and international orientation. Employees mainly work in teams on agile projects.

In alignment, employees mostly are placed in group offices with few single rooms. Almost everyone works with a laptop with about half of the workplaces having a second screen. The Company supports home offices in allowing remote sessions. Therefore, often times, computers are running at weekends and overnight. Further standard equipment consists of a telephone, which mostly is powered via network. Additional private devices are not allowed. Shared appliances include multifunctional printer stations, routers, a video- and an audio conferencing system. In the department's social rooms, there are dishwashers, fridges, coffee makers, kettles and boilers.

Overall, we provided 57 workplaces with a smart plug based sensor technology in bureaus accommodating between one and four employees. Where accessible, we additionally measured appliances in pooled and recreational areas. We did not cover HVAC and light controlling devices, since they were handled via building automation and therefore were not accessible to employee's behavioral choices.

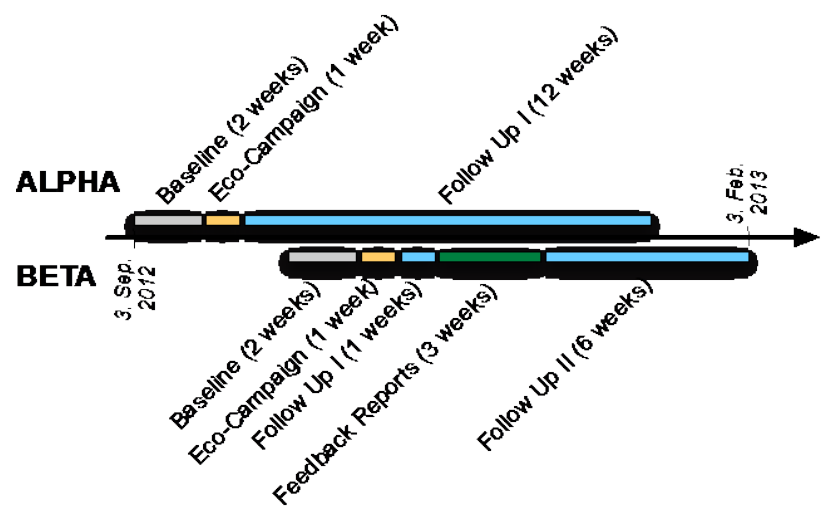

Figure 2: Intervention timeline in Alpha (top) and Beta (down)

\section{B. Company Beta}

Beta is a finance service provider with over 30 branch offices and headquarters including a service center and two administrational buildings. The company's culture is coined by high seriousness and solidity. As another characteristic, the company is firmly rooted regionally and shows strong colleagueship.

For the study, we chose a department within administration, mainly engaged in construction financing and architecture, consisting of 30 workplaces. Furthermore, there is a recreational room, featuring a fully equipped kitchen and a printing pool with four printers, a plotter, a scanner and a plan copier. Offices accommodate up to three employees. In case of beta, all of department's workplaces were included into our measurements. Standard equipment includes a cellular phone and base station. Almost everyone uses a personal computer and one flat screen monitor, with 18 participants having a fatclient (regular PC) and nine employees working on thinclients (virtual desktops). Exceptions represent few employees provided with a second screen for special purposes and three participants with two computers constituting the department's IT. Just as with Alpha, private appliances are not used regularly. Appliances in pooled and recreational areas were captured, too, while we excluded light and HVAC.

In both cases a main requirement, was that our interventions were not to interfere with everyday work, or cause extra workload for employees. For increasing acceptance on both, management and employee level, we therefore planned a persuasive, yet not intrusive energy campaign for each company. In return, we benefited from management support, where a great commitment existed throughout the whole study, e.g. in gaining access to internal communication systems for feedback provision.

Despite similarities, there were significant differences between both organizations' cultures as well as in existing information infrastructure. This also has consequences for the design of our information and motivation campaign as discussed below in more detail.

\section{INTERVENTION DESIGN AND REALIZATION}

For comparability of both studies we also attempted to keep a similar course and period of actions, where we spilt our intervention into different stages (cf. Figure 2) including a baseline measurement, a one-week information-campaign and two follow-up measurements (plus feedback reporting in the case of Beta).

In the first phase, we conducted a two-week baseline measurement, which in both companies was carried out in third quarter of 2012. Secondly, we mounted a one-week ecocampaign, which we adapted to each company's specific 
conditions. During the third phase we measured the effects after campaigning over a period of two weeks.

In Alpha, we recorded consumption for ten more weeks without any additional intervention. In Beta, we had the opportunity to provide additional personalized, weekly feedback reports for three weeks. Afterwards, we kept the sensors in place for seven more weeks until February 2013 to measure follow-up effects.

\section{A. Baseline}

The goal of baseline measurement was to provide status-quo data, therefore allowing comparison of changes in energy consumption in later phases with default values on appliance level.

In both companies, the staff association was informed about campaign's scope, installation of sensors and handling of sensitive data. In anticipation of privacy concerns, we proposed to let an internal employee of Beta create pseudonyms before processing and feeding back data. Additionally, we informed management utilities about our project, to comply with security regulations such as fire protection. Aiming for a baseline that would be as close as possible to normal usage, we avoided any unnecessary announcements. In Beta we only wrote an unobtrusive Email and introduced our study in an everyday jour fixe. Additionally, sensors sometimes were visible to participants.

\section{B. Information and Motivation Campaign}

Campaigning aimed at raising awareness of employees for saving energy at the workplace, motivating to contribute and communicating information on opportunities to save energy. On a conceptual level, we followed examples from literature $[8,21,23]$. Yet, it became evident that a theory-driven design could not be applied straight forward. Instead, we found that interventions and materials have to be adapted the particular organizational context and need to respect concerns of management and employees. For instance, while Alpha embraced the idea of using a sweep for motivating employees, Beta perceived this to be inappropriate and preferred motivating via hierarchical communication.

In addition, Alpha wanted the campaign to reflect its culture of a dynamic, innovative and international organization. As a consequence, an emphasis of the campaign was put on an experimental and adventurous characteristic, preferably using interactive media and modern English terms. During the campaign, every day informational materials of varying kinds and on different, established channels were distributed.

As core of measures for raising awareness, an information desk was positioned in the main buildings' foyer. This place offered high traffic especially around lunch breaks, where questions concerning energy consumption in both home and work environments were answered. At this desk, multi-plugs

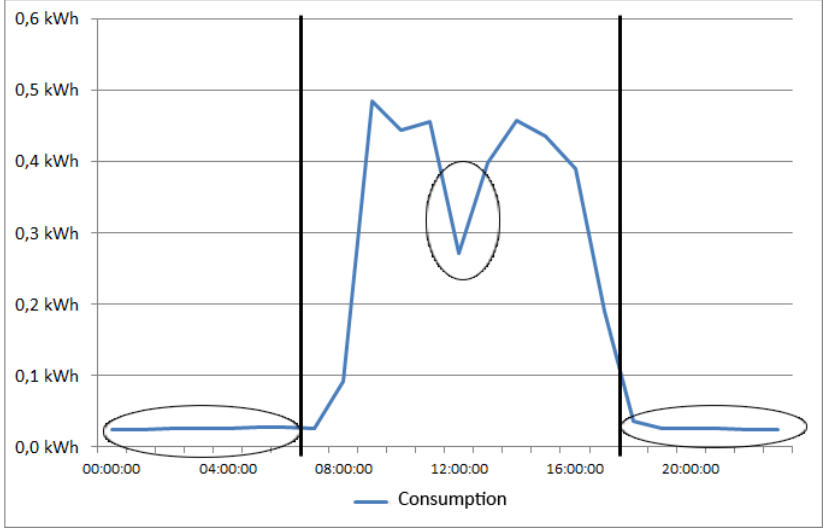

Figure 3: Characteristics consumption pattern showing when office worker in Beta come to work (left), going to lunch (center) and leaving the office (right).

with an external switch were offered, too, allowing workers to switch off all workplace devices easily. Employees were also able to participate in an "energy-challenge"-sweep. Furthermore, throughout the building, posters were applied, which on the one hand were supposed to raise awareness and motivate, while on the other hand served as a distributor of knowledge on problems and behavioral opportunities.

Up-to-date information was also provided via intranet and an interactive large-format screen in the foyer. Activities changed every day and included sending E-Mails, showing filmlets about sustainability initiatives in the company and their effectiveness, distributing postcards with energy saving hints and inviting to a talk on the topic. On the contrary, Beta wanted the campaign to be carried by its culture of seriousness and regional rootedness. Experimental aspects and English terms on information material were relinquished and German wording was used instead.

Since paper based information distribution is widely established in Beta, we fostered the use of media such as posters, postcards, stand-up displays and handouts. Interactive media were used only conservatively, focusing on spreading information rather than motivating directly with activities changing every day. For example, a comic-styled image-film shown at Alpha was not used for Beta. Instead we put emphasis on documents about energy saving potentials at home and at work.

As at Alpha, employees were able to approach an open information desk in a central place of the department, where all kinds of questions could be discussed.

\section{Individual Design of Feedback Solutions}

As a main benefit of smart metering technology, it allows consumers to receive feedback on individual consumption patterns in real-time. Making use of this advancement, we deployed Beta with high granular digital metering infrastructure in order to implement a comprehensive smart 
energy management system for employees, covering the whole process from data gathering, over processing to delivery. As there was no commercial system available that fulfilled all requirements of our partners, we developed an individualized reporting solution for personal feedback (cf. Figure 1). In addition, we created an anonymous bulletin report of aggregated consumption for the whole department. This report was hung out on the billboard and updated weekly.

Our design follows a pragmatic participatory oriented approach, where we take up design ideas from literature and discuss them with employees and management in informal talks as well as in formal meetings. This input provided us with further ideas to improve our design and what kind of information was important for them.

Our first sketches were informed by established eco-feedback design recommendations like keeping visualizations low in detail and focusing on main statements supporting the user in understanding consequences of behavior in terms of energy consumption [16,35].

The aim was to transfer main information such as total consumption of past week in kilowatt-hours, and percentage difference to previous week. Additionally, we included means for comparison among weekly consumption, supplemented with a weather metaphor and provided general information about peaks, lows and average. This metaphor (sun=decreased consumption, rain=increased consumption) takes up on the advice that user would profit from receiving laudatory and dispraising emotional feedback respectively, depending on development of consumption patterns $[17,28]$.

In discussing the issue with management, this was affirmed to be a pursued aim for Beta, too. We therefore added a second chapter to our feedback sheet, featuring more disaggregated information divided into working and non-working hours. We also talked over common strategies like goal setting and comparative feedback with our partner as they pose established strategies of motivation as well [2,14,27]. In particular, we recommended a leaderboard where office workers could compare their energy data against peers in the weekly reports. However, this was refused especially by Beta, since competitive elements would not fit into general culture and self-image of the company. Furthermore, individualized metering data was perceived as a private issue that belongs to each employee's informal self-determination (see also [33] for a similar observation). Therefore, we abandoned comparative elements in this study.

When discussing, how to provide meaningful information, it also became evident that wasting energy when not working is an important, yet neglected topic in eco-feedback research: Outside of main working hours, energy consumption should be entirely avoided. Based on characteristics of workplace energy consumption patterns, feedback systems should reflect this distinction [22,25,42].
By additionally showing this week's daily consumption compared to each day of the previous week, this chapter picks up on recommendations to provide historic comparison $[2,16,34]$, too.

Based on load-profiles (cf. Figure 3) measured during baseline period and talks with management, we broadly distinguished between $7 \mathrm{am}-6 \mathrm{pm}$ as working hours, $6 \mathrm{pm}$ to 7 am as nonproductive times and weekends. In future, we also plan to take public holidays into account. For this study, we used a timespan without national holidays included in our measurements.

The sheet was created based on gathered data in a semiautomatic fashion. For privacy reasons, records neither were passed on to colleagues, nor were they accessible to superiors at any time. Pseudonymization granted that only one man within Beta could allocate reports to each participant.

\section{RESULTS}

\section{A. Baseline Consumption in Both Companies}

Figure 4 presents the averaged weekly consumption of an office worker in Alpha and Beta during the two weeks of baseline determination.

In the two weeks of baseline period, Alphas 57 workplaces consumed $418.1 \mathrm{kWh}$ in total, while measuring $47.2 \mathrm{kWh}$ for collective appliances in pools and $122.2 \mathrm{kWh}$ in recreational rooms. The 30 workplaces of Beta consumed $268.3 \mathrm{kWh}$ in total, with collective appliances amounting to $27.8 \mathrm{kWh}$ and the recreational room consuming $12.4 \mathrm{kWh}$.

The pools and recreation rooms represent common costs. Therefore, in order to simplify the calculation of mean workplace consumption, we distributed shares equally between individual workers. However, this data can only serve as a broad orientation, since individual usage of collective appliance could not be accounted exactly (cf. Figure 4). In Alpha a worker's mean weekly consumption added up to 5.2 kWh (workplace: $3.7 \mathrm{kWh}$; prorated pool: $0.4 \mathrm{kWh}$; prorated recreation: $1.1 \mathrm{kWh}$ ). For Betas employees we got almost the same mean total consumption of $5.2 \mathrm{kWh}$ (workplace: 4.5 kWh; prorated pool: $0.5 \mathrm{kWh}$; prorated recreation: $0.2 \mathrm{kWh}$ ). Workplace consumption therefore differs by $21.6 \%$.

In contrast to other studies $[8,23,41]$ the installed smart sensors gave us the chance not only to measure aggregated consumption, but also to harvest fine-grained load profiles of office workers in a timely fashion. This electricity footprint allowed us to infer common work patterns such as times of arrival at work and lunchtime breaks (cf. Figure 3). For instance, Betas employees, with very small deviances, show up around 8 am and leave around $5 \mathrm{pm}$. These clearly defined hours are not caused by official directions, since employees have flexible schedules. Alpha showed even more loosely 
regulated working hours, with most employees coming between 7 and 9 am, and leaving between 4 and 6 pm.

Based on these patterns, we defined working hours to range from 8 am to $7 \mathrm{pm}$ for Alpha and 7 am to $6 \mathrm{pm}$ for Beta, which according to management were quite generous estimations, already. Further load profile analysis showed that non-working hours and the weekend account for $28.6 \%$ of Alphas total consumption (working hours: $3.7 \mathrm{kWh}$; nonworking hours: $1.0 \mathrm{kWh}$; weekends: $0.5 \mathrm{kWh}$ per employee). In case of Beta the percentage is even higher, amounting to 37.2\% (working hours: $3.2 \mathrm{kWh}$; non-productive hours: 1.2 kWh; weekends: $0.8 \mathrm{kWh}$ ).

For Alpha this high share could partially be explained by workers not switching off computers in order to work from home via remote access or for running complex test jobs over the weekend. For Beta though, employees as well as management were quite surprised about this finding, since working hours were estimated conservatively already. Moreover, in opposition to Alpha, the company had a quite strict firewall policy for security reasons, prohibiting any remote access. With no necessary usage known, there was no reason for consumption outside of working hours (on the upside, our finding revealed high energy saving potentials for Beta, to be tapped into simply by motivating workers to shut down appliances at closing-time).

\section{B. Effects of the Intervention on Consumption}

Figure 5 shows course of energy consumption over the weeks our study.

Concerning eco-campaign effects on energy conservation, the first follow-up unfolded average reductions of $4.2 \%$ in Alpha within in the next two weeks after providing information (in these two weeks, one day was official holiday that we excluded, and used the mean consumption of the week instead). Closer analysis revealed that average consumption in working hours almost did not change $(+0.1 \%)$ while total savings largely stemmed from reductions during nonproductive hours (-17.2\%) and weekends (-9.9\%). Obviously, behavioral change mainly related to switching off devices, when leaving the office after work.

Disaggregating data to device level additionally shows that both, consumption of personal equipment $(-5.9 \%)$ and of recreational appliances $(-13.0 \%)$ dropped, while pooled devices increased by 33.3\%. Campaigning obviously helped to motivate participants to switch off their devices at the desk, while not showing the same result concerning shared appliances.

From the third to sixth week after campaigning, total consumption increased by $2.6 \%$ in average compared to the baseline period. The disaggregation reveals that this resulted from a raise during working hours $(+8.1 \%)$, while the positive effects in non-productive hours $(-13.4 \%)$ and weekends ($6.6 \%)$ still existed.

The first week after the campaign in Beta also shows that savings were mainly achieved outside working hours. In total, the department's consumption was reduced by $13.3 \%$, with savings during working hours $(-7.5 \%)$ turning out lower than the significant changes in non-productive times $(-20.8 \%)$ and on weekends $(-26.7 \%)$. On device level reductions could be noted at the workplace $(-9.5 \%)$, but especially pooled consumption was significantly reduced by $56.2 \%$. This was mainly achieved by unplugging a seldom used large printer. Among recreational devices, usage stagnated (+0.6\%).

In the period of the subsequent personalized feedback provision from fourth to seventh week, average energy consumption was $27.1 \%$ lower than in the baseline period (working hours: -19.9\%; non-productive hours: $-37.2 \%$; weekends: -42.6\%). Asking management for possible blind spots, holiday plans and cases of illness showed no anomalies. Rather, management told that typically before Christmas even more work has to be done.
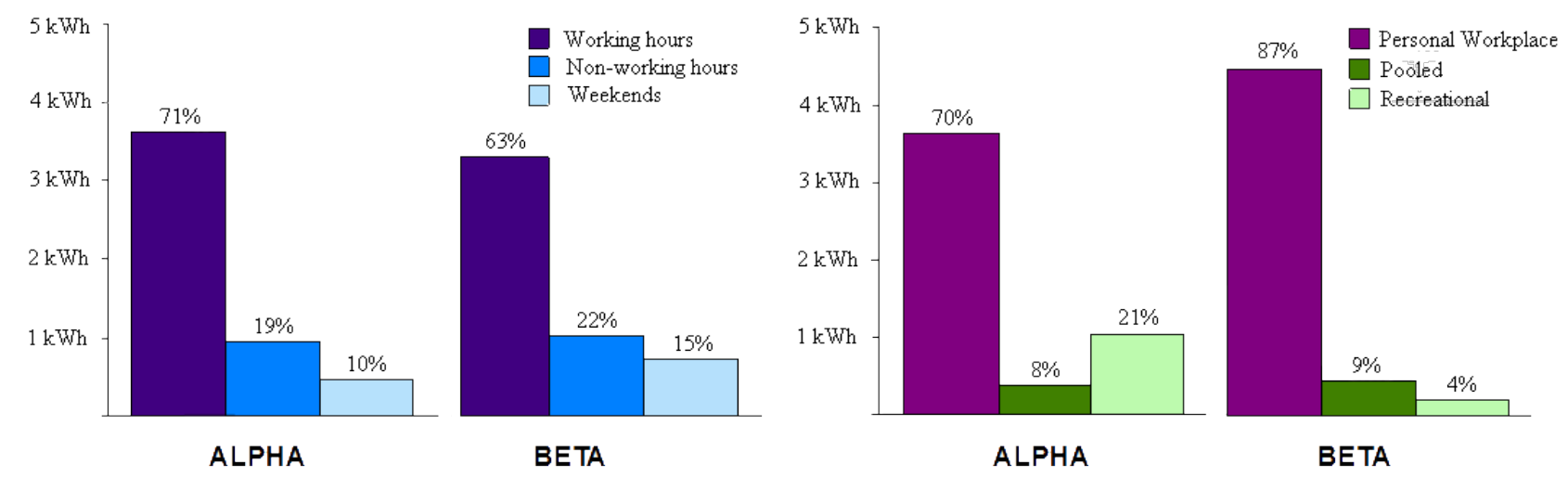

Figure 4: Averaged weekly consumption of an office worker during baseline period (left: disaggregated by time; right: disaggregated by device type) 
order to study follow-up behavior, we excluded weeks between Christmas and New Year's because of holidays. Instead, we have calculated the average consumption from 14th of January until the February 3rd (seven weeks after the last feedback report). In this period, the total saving decline, but still $12.2 \%$ could be conserved compared to baseline period. This was mainly archived outside of working hours (non-productive hours: -27.0\%; weekends: -29.1\%). A small reduction of $3 \%$ was remained in working hours between $7 \mathrm{am}$ and $6 \mathrm{pm}$. Overall, the decline of conservation, however, indicates that after the holiday break some old "bad" habits creep back into everyday lives of participants.

\section{Findings from talks with participants}

After the follow-up measures, we conducted open talks with five employees of Beta concerning the intervention, asking about their behavioral change, taken measures to save energy and their ideas to improve eco-campaigning in future. On request of the employees, these talks were not recorded. In addition, in Alpha a three-hour workshop was conducted which was attended by one manager, three researchers and eleven employees. This workshop reflected the "lessons learned" and included a discussion about options to improve eco-interventions in the future.

Concerning motivation, in Alphas employees told us that they had a high motivation right from the beginning of the study. This was expected to some extent, as the company is a specialized sustainability-IT provider. Yet, in case of Beta, employees at first had a more neutral to skeptical attitude, despite mostly being generally interested in energy saving at home. This initial skepticism, however, changed during the pilot as many participants were surprised by consumption information. In particular, becoming aware of general high consumption during non-working hours, led to a higher interest in energy saving potentials at one's own workplace, too. Also, the opportunity to lend a set of smart-plugs to measure domestic energy consumption was well received. In particular, employees got familiar with eco-feedback

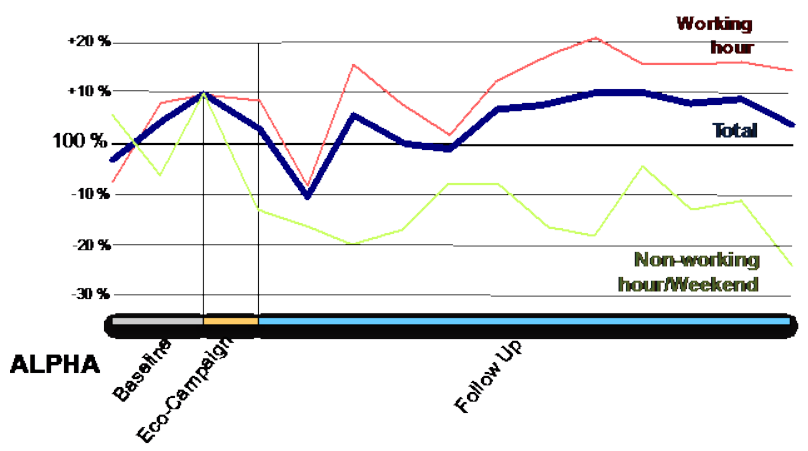

technology, which not just increased motivation and energy awareness at home, but also had a spill-over effect on trying to save energy at work, we were told.

Concerning the design of materials provided, we received largely positive feedback, with potential for optimization in detail. For instance, the personalized weekly reports were considered helpful for the direct feedback on personal behavior. In the financial context of Beta, employees emphasized the usefulness of money as the major feedback unit. The feedback of the reached conservation, however, was criticized as too simple. In particular, it does not consider events like appointments outside of the office or compelled to do some extra work. Therefore, instead of getting a special praise, our report fed back a rainy cloud, for consuming more energy than usual. This was judged to be misleading and unfair.

At Alphas workshop, a quite controversial topic was the use of behavioral versus technological measures for optimizing energy consumption. In particular, for people of the IT department "pure" motivational measures had the drawback of depending on the goodwill of employees, thus being prone for fallbacks into old unsustainable habits. Instead, they promoted automatic, but sophisticated customizable power management. In opposition, some workers argued that such solutions are already in place and have found to be insufficient for capturing the situated work context like ad-hoc late hours. In addition, existing configuration options are not known or too complicated to handle comfortably. The last point includes the fact that both, technical, and changes in organization must be reported to the IT department, for energy automation to be adapted. Yet there was a consensus that lightweight solutions like the switchable multi-plugs are a good strategy for helping people to sustain their pro-environmental behavior.

\section{DISCUSSION}

In summary, the studies demonstrate that behaviorally oriented measures used in domestic context [2,10,11,16]

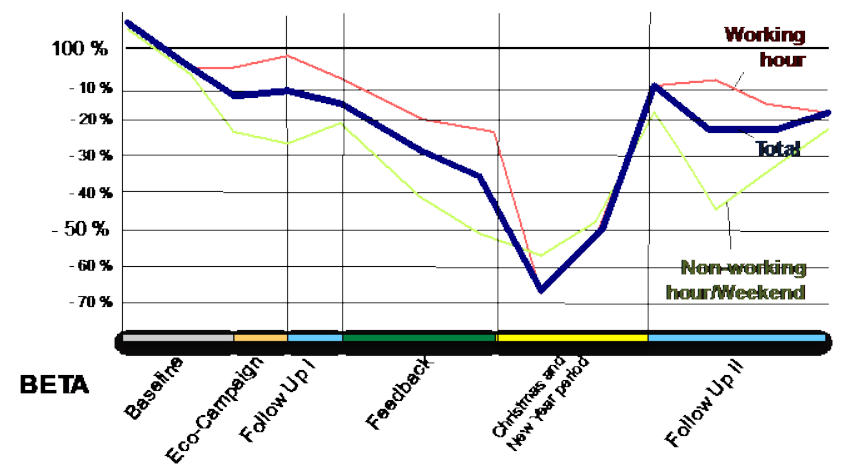

Figure 5: Changes of energy consumption all over the intervention at Alpha (left) and Beta (right) 
generally prove to be quite promising in the office setting, too and thus is in line with other studies[8,33].

However, in terms of energy consumption, there are significant differences between domestic and organizational context. We found how self-images of organizations and their culture had a strong influence on our ways of conduct. Furthermore, our findings suggest dimensions of meaning of energy consumption that help workers making energy accountable at the workplace that differ from home context. Finally, we show how our sustainability-efforts were successful, but remained a one-time intervention declining long-term effects.

In the following we discuss some of these topics in more detail, helping to transfer existing concepts and findings to organizational context.

\section{A. Taking the Organizational Context into Account}

Eco-feedback design for the workplace is a relative recent topic in HCI. Hence, it is honorable to uncover common requirements and identify canonical design issues [4,15,44]. Yet, as noted by He et al. [19] one size does not fit all. While the original remark refers to people's diverse motivation, our study shows that it also holds with regard to heterogeneity of organizational settings. We found several factors crucial for the study to be allowed by organizations' management in the first place.

Firstly, there were juridical necessities such as informing staff association about measuring energy consumption and maintenance about installing smart plug sensors in the fore field. These were mandatory activities, we largely expected. This demonstrates that design must take matters of privacy into account when measuring energy data at the workplace (see also [33]). Secondly however, during planning, we found how organizations' self-image had a strong impact when it came to planning interventions, too. Though we conducted collective planning workshops with both companies involved, measures developed in different ways: For instance, for Alphas culture a motivational campaign was perceived suitable, while Betas management refused such a coining and demanded stronger focus on businesslike information. While competitive persuasive approaches (like social comparison or goal setting) have proven successful in many cases (mainly shown in domestic settings) $[2,14,16,27]$ it was perceived as inappropriate for Beta. Therefore, the design form, style and use of emotions had to be adapted to the particular organizational corporate design, existing communication channels, technical infrastructure and interests of the user group, as certain elements were considered to not fit into the collegial culture by each company's management. This reveals, that in addition to competitively oriented approaches, collegial approaches for energy saving should be investigated into in the future. As described, we could not use the off-shelf reporting tool of the used smart plugs as it was not adaptable to demands concerning cultural requirements, privacy and access control of the participating organizations.

\section{B. Making Wasting Accountable}

Recent smart metering technology allows to match known demand [11,16] for fine-grained, direct feedback. Based on our experience, we would argue that the mere amount of unfiltered data and its richness in detail might even be considered harmful, when fed back without sufficient selection, as it might easily lead to an information overflow. Hence, instead of feeding back raw data in real time, ecofeedback should provide the right information in the right place at the right time. Concerning this, fine-grained gathering has a two-folded advantage: it helps researchers to analyze existing behavior pattern and to quantify saving potential in detail. By the same token, providing the right feedback helps to provide target-oriented information and motivation.

In accordance with other studies [22,25,41], our findings show significant consumption to occur outside of working hours (Alpha: 28.6\%; Beta: 37.1\%) and leaving the office to probably be the most critical event for decisions on wasting or saving energy. Although a well-known fact, it seems we are the first to address this consideration in eco-feedback design by distinguishing between working and non-working hours. The savings achieved as well as the positive comments of our participants make us argue, that giving the right feedback and making wasting accountable can provide a great benefit.

In detail, however, our user's comments also show that our solution was too simple, as it does not take e.g. vacations or extra time into account. Therefore, an essential future topic is to develop more sophisticated methods for more precise analysis and feedback provision. Campaigns would benefit, for example, from better understanding of systematic behaviour change and its connected effects, while filtering out and compensating the "noise" of particular special events (e.g. holidays, illness, and appointments away from the office). These improvements are equally demanded by eco-feedback researchers, designers and users. Moreover, our study shows that declaration of consumption to be waste is highly dependent on context and the local actors notion of the consumed services [35,40]. Hence, algorithms and concepts of visualization should be refined in participation of diverse stakeholders. This especially holds for the open topic, of appropriately prorating consumption of shared appliances.

\section{Making Conservation Sustainable}

In both cases, we observed energy conservation declining after a while (where Beta had a slightly steeper decline than Alpha). As in this time context remained largely unchanged, it seems that employees started to fall back into old "bad" habits successively. A similar phenomena is noted by Blevis [6], while Azar and Menassa [4] provide a counter example. In domestic context, some research $[5,10,18]$ notes similar phenomena with effects of eco-feedback declining over a 
longer period. Remarkably, Alpha outperforms Beta in this regard, especially during non-working hours. Further research investigating into reasons for these differences should be done. One explanation might be that multi-plugs distributed in Alpha made it easier for the office workers to switch off when leaving the desk.

This shows a further challenge to sustaining behavioral changes when designing smarter feedback solutions. Regarding this, Corradi et al. [9] suppose reducing the cognitive demands of energy saving behaviors. In addition, we suppose that energy saving should become part of the routinized, socio-material practices [32]. A promising, yet under-investigated approach in this direction presents the fusion of both, technological and behavioral oriented concepts. In particular, getting the user in the loop of automated power management could help to increase their usability and user acceptance. Complementary, smart eco-assistant systems could help to relieve people from tedious sustainability-work like checking that all devices are turned off at closing time.

\section{LIMITATIONS}

Due to the small sample size and the relatively short measurement period, our study could indeed give a first impression, but could not claim any statistical representativeness. In addition, Figure 5 shows that not only campaigning itself, but also even announcing an intervention and installing sensors had positive effects on energy behavior among participants. This is why actual baseline would be somewhat higher than the one we captured. Thus, savings should be rated slightly higher as well.

Another general limitation is that (to the best of our knowledge) there is no generally accepted procedure for dealing with seasonal effects, holidays, extra hours. Handling such events consistently is necessary in order to transparently and comparably estimate effects of behavioral change and separate them from perturbations. Murtagh et al. [25], for instance, did not take them into account at all to avoid distortion of the measured data, thus bearing the risk to draw false conclusion (e.g. stating a behavioral change, while it mainly a vacation effect). In our study, we therefore checked that our data is consistent in terms of amounts of employees taking holidays or being off sick. In addition we treated official holidays as non-working days and used mean consumption of the four-day-week instead. During intervention there in Alpha we carry out data preparation for the German National Holiday (10/3/12) and All Hallows Day (11/1/13). The latter was a bridging day, too (as the saving was unusually high, we excluded this day from our calculation). For Beta, we had no apparent holidays, but we excluded the vacation time around Christmas and New Year`s from our calculation. Another data preparation was needed in Alpha on the very first day of baseline measurement as for technical reasons our sensors have not captured the whole day.

\section{CONCLUSION}

Sustainable HCI, so far, has mainly focused on domestic context. Our study demonstrates that it is worth to investigate in behavior-based interventions at the workplace, too. As our results shows, the consumption could be reduced between $5 \%$ and $12 \%$ in both cases just by behavioral change. We additionally have demonstrated how knowledge regarding the design of energy feedback for workplaces does not have to start on a clean sheet, but that existing design guidelines for energy feedback in the home can successfully be transferred to workplace environments to some extent. It also reveals that specific organizational factors, such as management support, compliance with privacy and security demands, and respecting the organizational context are important factors for securing the studies' acceptance with both, the organization and the employees as well.

Because of the small number of empirical studies on sustainable HCI in the work context, however, we want to validate our results in further studies, in order to build an empirically driven framework for flexible smart energy management and feedback in organizations.

\section{REFERENCES}

[1] Abrahamse, W., Steg, L., Vlek, C., and Rothengatter, T. A review of intervention studies aimed at household energy conservation. Journal of Environmental Psychology 25, 3 (2005), 273-291.

[2] A Greening, L., Greene, D.L., and Difiglio, C. Energy efficiency and consumption-the rebound effect-a survey. Energy policy 28, 6 (2000), 389-401.

[3] Ardehali, M.M. and Smith, T.F. Evaluation of HVAC system operational strategies for commercial buildings. Energy Conversion and Management 38, 3 (1997), 225-236.

[4] Azar, E. and Menassa, C.C. A comprehensive analysis of the impact of occupancy parameters in energy simulation of office buildings. Energy and Buildings 55, (2012), 841-853.

[5] Bartiaux, F. Does environmental information overcome practice compartmentalisation and change consumers' behaviours? Journal of Cleaner Production 16, 11 (2008), 1170-1180.

[6] Blevis, E. Sustainable interaction design: invention \& disposal, renewal \& reuse. Proceedings of the SIGCHI conference on Human ..., (2007), 503-512.

[7] Brandon, G. and Lewis, A. Reducing household energy consumption: a qualitative and quantitative field study. Journal of Environmental Psychology 19, 1 (1999), 75-85.

[8] Carrico, A.R. and Riemer, M. Motivating energy conservation in the workplace: An evaluation of the use of group-level feedback and peer education. Journal of Environmental Psychology 31, 1 (2011), 1-13.

[9] Corradi, N., Priftis, K., Jacucci, G., and Gamberini, L. Oops, I forgot the light on! The cognitive mechanisms supporting the execution of energy saving behaviors. Journal of Economic Psychology, (2012).

[10] Van Dam, S.S., Bakker, C.A., and Van Hal, J.D.M. Home energy monitors: impact over the medium-term. Building Research \& Information 38, 5 (2010), 458-469.

[11] Darby, S. The effectiveness of feedback on energy consumption. A Review for DEFRA of the Literature on Metering, Billing and Direct Displays. Environmental Change Institute, University of Oxford, Oxford, 2006.

[12] DiSalvo, C., Sengers, P., and Brynjarsdóttir, H. Mapping the landscape of sustainable HCI. Proceedings of the 28th international conference on Human factors in computing systems - CHI '10, (2010), 1975. 
[13] 13. Dourish, P. Points of Persuasion: Strategic Essentialism and Environmental Sustainability. Persuasive Pervasive Technology and Environmental Sustainability, Workshop at Pervasive, Citeseer (2008).

[14] 14. Foster, D., Lawson, S., Blythe, M., and Cairns, P. Wattsup?: motivating reductions in domestic energy consumption using social networks. Proceedings of the 6th Nordic Conference on HumanComputer Interaction: Extending Boundaries, ACM (2010), 178-187.

[15] Foster, D., Lawson, S., Wardman, J., Blythe, M., and Linehan, C. Watts in it for me?: design implications for implementing effective energy interventions in organisations. Proceedings of the 2012 ACM annual conference on Human Factors in Computing Systems, ACM (2012), 2357-2366.

[16] Froehlich, J., Findlater, L., and Landay, J. The design of eco-feedback technology. Proceedings of the 28th international conference on Human factors in computing systems - CHI '10, ACM Press (2010), 1999-2008.

[17] Gustafsson, A. and Gyllenswärd, M. The power-aware cord: energy awareness through ambient information display. CHI'05 extended abstracts on Human factors in computing systems, ACM (2005), 14231426.

[18] Hazas, M., Brush, A.J., and Scott, J. Sustainability does not begin with the individual. interactions 19, 5 (2012), 14-17.

[19] He, H.A., Greenberg, S., and Huang, E.M. One size does not fit all: applying the transtheoretical model to energy feedback technology design. Proceedings of the 28th international conference on Human factors in computing systems, ACM (2010), 927-936.

[20] Jackson, T. Motivating sustainable consumption. A review of evidence on consumer behaviour and behavioural change. A report to the Sustainable Development Research Network, Surrey: Centre for Environmental Strategies, January (2005).

[21] Kastner, I. and Matthies, E. Implementing web-based interventions to promote energy efficient behavior at organizations - a multi-level challenge. Journal of Cleaner Production, (2013).

[22] Masoso, O.T. and Grobler, L.J. The dark side of occupants' behaviour on building energy use. Energy and Buildings 42, 2 (2010), 173-177.

[23] Matthies and Wagner. Change-Veränderung nachhaltigkeitsrelevanter Routinen in Organisationen. LIT Verlag Münster, 2011.

[24] Melville, N.P. Information systems innovation for environmental sustainability. MIS Quarterly 34, 1 (2010), 1-21.

[25] Murtagh, N., Nati, M., Headley, W.R., et al. Individual energy use and feedback in an office setting: A field trial. Energy Policy, 62 (2013), 717-728.

[26] Murugesan, S. Harnessing green IT: Principles and practices. IT professional 10, 1 (2008), 24-33.

[27] Petkov, P., Köbler, F., Foth, M., and Krcmar, H. Motivating domestic energy conservation through comparative, community-based feedback in mobile and social media. Proceedings of the 5th International Conference on Communities and Technologies, ACM (2011), 21-30.

[28] Pierce, J., Odom, W., and Blevis, E. Energy aware dwelling: a critical survey of interaction design for eco-visualizations. ... Interaction: Designing for Habitus and ..., (2008).
[29] Pierce, J. and Paulos, E. Materializing energy. Proceedings of the 8th ACM Conference on Designing Interactive Systems, (2010), 113.

[30] Pierce, J., Schiano, D.J., and Paulos, E. Home, habits, and energy: examining domestic interactions and energy consumption. Proceedings of the SIGCHI Conference on Human Factors in Computing Systems, ACM (2010), 1985-1994.

[31] Poortinga, W., Steg, L., Vlek, C., and Wiersma, G. Household preferences for energy-saving measures: A conjoint analysis. Journal of Economic Psychology 24, 1 (2003), 49-64.

[32] Reckwitz, A. Toward a Theory of Social Practices: A Development in Culturalist Theorizing. European journal of social theory 2002, 2 (2002), 245-265.

[33] Schwartz, T., Betz, M., Ramirez, L., and Stevens, G. Sustainable energy practices at work. Proceedings of the 6th Nordic Conference on HumanComputer Interaction Extending Boundaries - NordiCHI '10, ACM Press (2010), 452-462.

[34] Schwartz, T., Denef, S., Stevens, G., Ramirez, L., and Wulf, V. Cultivating energy literacy: results from a longitudinal living lab study of a home energy management system. Proceedings of the SIGCHI Conference on Human Factors in Computing Systems, (2013), 11931202.

[35] Schwartz, T., Stevens, G., Ramirez, L., and Wulf, V. Uncovering practices of making energy consumption accountable. ACM Transactions on Computer-Human Interaction 20, 2 (2013), 1-30.

[36] Siero, F.W., Bakker, A.B., Dekker, G.B., and van den Burg, M.T. Changing organizational energy consumption behaviour through comparative feedback. Journal of Environmental Psychology 16, 3 (1996), 235-246.

[37] Spagnolli, A., Corradi, N., Gamberini, L., et al. Eco-feedback on the go: Motivating energy awareness. Computer 44, 5 (2011), 38-45.

[38] Stern, P.C. New Environmental Theories: Toward a Coherent Theory of Environmentally Significant Behavior. Journal of Social Issues 56, 3 (2000), 407-424.

[39] Stern, P.C. and Aronson, E. Energy Use: The Human Dimension. W.H. Freeman \& Company, 1984.

[40] Strengers, Y.A. Designing eco-feedback systems for everyday life. Proceedings of the SIGCHI Conference on Human Factors in Computing Systems, ACM (2011), 2135-2144.

[41] Webber, C.A., Roberson, J.A., McWhinney, M.C., Brown, R.E., Pinckard, M.J., and Busch, J.F. After-hours power status of office equipment in the USA. Energy 31, 14 (2006), 2823-2838.

[42] Wong, J.K.W., Li, H., and Wang, S.W. Intelligent building research: a review. Automation in Construction 14, 1 (2005), 143-159.

[43] Yun, R., Lasternas, B., Aziz, A., et al. Toward the design of a dashboard to promote environmentally sustainable behavior among office workers. Proceeding PERSUASIVE'13 Proceedings of the 8th international conference on Persuasive Technology, (2013), 246-252.

[44] Yun, R., Scupelli, P., Aziz, A., and Loftness, V. Sustainability in the workplace: nine intervention techniques for behavior change. In Persuasive Technology. Springer, 2013, 253-265. 